\title{
Desinstitucionalización y calidad de vida en pacientes esquizofrénicos. El caso de Segovia
}

RESUMEN: Estudio, veinte años después de la reforma psiquiátrica, con el que pretendemos dar a conocer, cómo es la calidad de vida en pacientes con esquizofrenia.

PALABRAS CLAVE: Esquizofrenia, lugar de residencia, estado funcional, calidad de vida subjetiva y objetivada.
SUMMARY: Paper, based on a study carried out two decades after the Psychiatric Reform in Spain, focuses on quality of life among schizophrenic patients.

KEY WORDS: Schizophrenia, place of residence, functional status, subjective vs. objetivized quality of life.

\section{Introducción}

La reforma psiquiátrica en abril de 1985, hizo que muchas personas que estaban ingresadas en Hospitales Psiquiátricos pasaran a recibir sus cuidados dentro del Sistema General de Salud. La medida permitía que este colectivo volviera a la Comunidad y se formularan unos criterios generales de prestación de asistencia a los problemas de salud mental (1).

Estos criterios se establecieron sobre la base de plena integración en el sistema sanitario general de todas las actuaciones relativas a dicha asistencia; la potenciación de los recursos asistenciales a nivel comunitario; la equiparación del enfermo mental a otros pacientes; la incorporación de las unidades psiquiátricas en los hospitales generales; el desarrollo de servicios de rehabilitación y reinserción, y la puesta en marcha de actividades preventivas en coordinación con los Servicios Sociales (2).

Con el presente estudio pretendemos dar a conocer, veinte años después de la reforma, cómo es la calidad de vida en pacientes esquizofrénicos de Segovia; realizando para ello una exposición comparativa entre los que actualmente viven institucionalizados en unidad residencial psiquiátrica y los que residen en la provincia o en la capital.

El concepto calidad de vida es de difícil precisión y ha dado lugar a diferentes modelos teóricos (3), que han generado a su vez, múltiples instrumentos de evaluación. Aunque no existe un consenso en cuanto a su definición, sí encontramos algunas características comunes. Este concepto debe incluir, por un lado, indicadores objetivos sobre una amplia variedad de aspectos de la vida y por otro debe incluir elementos subjetivos que valoren el grado de satisfacción o bienestar alcanzado por las personas, partiendo de su propia percepción. La incorporación de la subjetividad al constructo de calidad de vida se realiza 
a partir de Lehman en 1982 y se le ha dado una importancia creciente $(4 ; 5 ; 6)$. Teniendo en cuenta estas consideraciones, elegimos como instrumento especifico para medir la calidad de vida en esquizofrenia, el LQoLP, esto es, Perfil de Calidad de Vida de Lancashire.

En estudios anteriores, como el de Leisse y Kallert (7), la satisfacción subjetiva de los afectados, no difería sistemáticamente dependiendo del entorno de atención (hecho que corroboraremos en el nuestro). Los que vivían en instituciones, presentaban un grado relativamente alto de satisfacción subjetiva, a pesar de sus niveles mayores de discapacidad (teoría del nivel de adaptación, logro de metas y fenómeno de contraste). Björkman y Hansson (8), encontraron que el funcionamiento psicosocial, los síntomas psiquiátricos y la autoestima fueron los factores más importantes como predictores de calidad subjetiva de vida.

Nos decantamos también por introducir un doble criterio de valoración. No sólo se trata de conocer la percepción personal, sino que además, es necesario conocer de qué manera el paciente puede desarrollar su vida en la sociedad en que vivimos (9). Por supuesto, no se pretende establecer una mayor consideración por uno u otro método de medición. Creemos que ambos enfoques se complementan y son necesarios. Nuestro estudio pretende también identificar las variables que podrían incidir en la calidad de vida, tanto subjetiva como objetiva.

\section{Método}

a) Participantes y ámbito del estudio

Participaron en el estudio 61 pacientes de entre 45 y 60 años con diagnóstico de Esquizofrenia según CIE 10: F20 (10), residentes en Segovia. El límite de edad se decidió pensando en acotar una población joven en las fechas de inicio de la reforma. Se seleccionaron aquellos que hubieran ingresado a lo largo de su vida en la Unidad de Hospitalización Psiquiátrica o bien se encontraran en la actualidad en la Unidad de Rehabilitación «Nuestra Señora de la Fuencisla» ${ }^{1}$. Pretendíamos incluir de esta manera a los enfermos con cuadro clínico más grave y de edad joven en las fechas del inicio de la reforma, para tener información sobre la evolución de su enfermedad a largo plazo.

1 Unidad indicada para pacientes carentes de autonomía social y personal. Cumple funciones esencialmente de carácter residencial y proporciona cuidados mínimos de enfermería para quienes la precisen. 
b) Instrumentos de medida

- Los datos sociodemográficos se recogieron por medio de un cuestionario de elaboración propia.

- Para valorar el estado funcional del paciente, utilizamos la Escala de Evaluación de la Actividad Global (EEAG), Eje V en el DSM-IV-TR (11).

- La calidad de vida se midió utilizando el instrumento: Perfil de Calidad de Vida de Lancashire Versión Europea (LQOLP- EV) $(12 ; 13)$. Se trata de una entrevista estructurada, que debe ser administrada por entrevistadores preparados (en nuestro caso, profesionales de los Servicios de Salud Mental) en un tiempo aproximado de 30 minutos. Consta de 105 elementos que evalúan el bienestar global de la persona, su autoconcepto, así como el bienestar, en nueve áreas principales de la vida tanto desde una perspectiva subjetiva como objetiva: Trabajo/educación, tiempo libre/participación de actividades recreativas, religión, finanzas, alojamiento, problemas legales y de seguridad ciudadana, relaciones familiares, relaciones sociales, salud. Los componentes subjetivos de estos dominios son evaluados en una Escala de 7 puntos de Satisfacción Vital (ESV) (1: no puede ser peor, hasta 7: no puede ser mejor). El cuestionario incluye 2 escalas para medir el estado afectivo y la autoestima del paciente. Así como una valoración por parte del entrevistador de la calidad de vida percibida del sujeto (Uniescala). Este instrumento fue validado y traducido al español por Luis Gaite y colaboradores en el estudio EPSILON 8 (14).

\section{c) Análisis de los datos}

Las técnicas estadísticas empleadas se resumen en: descriptivos, ANOVA, Prueba de Kruskal-Wallis, ambas con análisis post-hoc (Scheffé y Steel, en su caso), Prueba T, Prueba U de Mann-Whitney (junto con la K W, según escala o por incumplimiento de la hipótesis de homogeneidad de la varianza), Prueba $\chi^{2}$ de Pearson (recurriendo a los residuos tipificados corregidos), Coeficiente de correlación de Pearson mediante Kyplot 2.0 (Beta) (15) y SPSS 11. En todos los contrastes, la significatividad se marca con un nivel $\alpha$ máximo del $5 \%$.

\section{Resultados}

1) Información sociodemográfica y clínica:

De los 61 pacientes que participaron en el estudio, el 66\% eran hombres y el 34\% mujeres. Con una edad comprendida entre los 45-60 años, siendo la 
media $52( \pm 4)$. Al comprobar la distribución por lugar de residencia, el $31 \%$ residen en la Unidad de Rehabilitación, mientras que un 23\% viven en Segovia capital y un $46 \%$ en la provincia (31\% institucionalizados vs $69 \%$ no institucionalizados). Queríamos hacer especial hincapié en la dependencia o no dependencia institucional de los pacientes. La división entre provincia y capital se hace para identificar si existen además peculiaridades en los enfermos residentes en ambos entornos. Así, definimos tres entornos distintos de residencia (Tabla 1).

La diferencia de edades no es significativa en ninguno de los tres grupos establecidos por lugar de residencia. El mayor número de mujeres se concentra en la Unidad de Rehabilitación (57\% vs $17 \%$ de los hombres) y el mayor número de hombres en la provincia (50\% vs $38 \%$ de las mujeres). La Prueba de jicuadrado de Pearson en este sentido arroja un valor $\chi^{2}=12$; gl. $=2$; sig. $=$ $0,002^{2}$.

En cuanto a la distribución por diagnósticos, el 29\% de los pacientes fueron diagnosticados de esquizofrenia residual. El 54\% de E. paranoide. El 17\% restante se reparte entre la simple, la hebefrénica y sin especificar (se recodifica la variable en tres niveles para los cálculos). La duración media de evolución de la enfermedad es de 22 años $( \pm 10)$.

Los pacientes tienen un Estado funcional medio (Escala de Evaluación de la Actividad Global, EEAG) de $56( \pm 16)$, situándose en el rango 51-60, es decir, síntomas moderados o dificultades moderadas en la actividad social y laboral. De los que presentan un estado funcional por debajo de 40 (alteración importante en varias áreas como el trabajo, las relaciones familiares, el juicio, pensamiento y estado de ánimo) el 60\% residen en la Unidad de Rehabilitación, repartiéndose la totalidad de los que presentan un estado funcional por encima de 60 (síntomas moderados), entre capital y provincia. Esto puede explicarse bien porque el paciente, tras años de evolución de su enfermedad, va reduciendo paulatinamente su estado funcional hasta que no puede vivir de forma autónoma o con sus cuidadores habituales, teniendo que ingresar entonces en unidad residencial, o bien porque la dependencia institucional tiende a reducir su estado funcional.

2 La diferencia (positiva) es significativa entre la frecuencia observada y la teórica, en el caso de los residentes en la Unidad de Rehabilitación para el sexo femenino y en el caso de la capital para los hombres. 
ORIGINALES Y REVISIONES

Tabla 1

Distribución en variables sociodemográficas por lugar de residencia (\%)

\begin{tabular}{|c|c|c|c|c|c|}
\hline & & & $\begin{array}{l}\text { Unidad } \\
\text { Rehabilitación }\end{array}$ & Capital & Provincia \\
\hline & Niveles & Número & $\%$ & $\%$ & $\%$ \\
\hline \multirow{2}{*}{ Sexo } & Hombre & 40 & 17,50 & 32,50 & 50,00 \\
\hline & Mujer & 21 & 57,14 & 4,76 & 38,10 \\
\hline \multirow{3}{*}{ Diagnóstico } & Residual & 18 & 50,00 & 27,78 & 22,22 \\
\hline & Paranoide & 33 & 15,15 & 21,21 & 63,64 \\
\hline & Otras & 10 & 50,00 & 20,00 & 30,00 \\
\hline \multirow{4}{*}{ Estudios } & Sin estudios & 1 & & & 100,00 \\
\hline & Primarios & 35 & 31,43 & 22,86 & 45,71 \\
\hline & Medios & 17 & 29,41 & 17,65 & 52,94 \\
\hline & Superiores & 8 & 37,50 & 37,50 & 25,00 \\
\hline \multirow{2}{*}{ Vive solo } & Sí & 10 & & 40,00 & 60,00 \\
\hline & No & 51 & 37,25 & 19,61 & 43,14 \\
\hline \multirow{3}{*}{$\begin{array}{l}\text { Estado } \\
\text { funcional }\end{array}$} & $<40$ & 20 & 60,00 & 5,00 & 35,00 \\
\hline & $40-60$ & 25 & 28,00 & 24,00 & 48,00 \\
\hline & $>60$ & 16 & & 43,75 & 56,25 \\
\hline \multirow{3}{*}{$\begin{array}{l}\text { Años } \\
\text { evolución } \\
\text { enfermedad }\end{array}$} & $<20$ & 21 & 33,33 & 19,05 & 47,62 \\
\hline & $20-30$ & 20 & 50,00 & 25,00 & 25,00 \\
\hline & $>30$ & 20 & 10,00 & 25,00 & 65,00 \\
\hline \multirow{4}{*}{ Estado civil } & Casado & 6 & 16,67 & 33,33 & 50,00 \\
\hline & Soltero & 49 & 30,61 & 22,45 & 46,94 \\
\hline & Divorciado & 4 & 50,00 & & 50,00 \\
\hline & Separado & 2 & 50,00 & 50,00 & \\
\hline
\end{tabular}

Estado funcional y años de evolución de la enfermedad presentan un coeficiente de correlación de Pearson -0,014; sig bilateral =0,92. Asimismo, el número en años medio de evolución de la enfermedad no es significativamente distinto por lugar de residencia. Es decir, los pacientes de la Unidad de Rehabilitación no presentan mayor número de años en la evolución de su enfermedad que el resto.

Sin embargo, sí existe diferencia contrastada en la media del estado funcional (Tabla 2) de los pacientes ingresados en la Unidad de Rehabilitación: ANOVA 2 factores $^{3}$ (lugar-diagnóstico) $\mathrm{F}=13$; gl. 2, 52; sig. < 0,001 para el primero (lugar

3 Nos interesaba conocer el efecto de la dependencia institucional en combinación con el diagnóstico. Si en el diagnóstico no era significativo pero en la interacción sí, podría corroborarse que el entorno del paciente influía en su estado funcional. 
de residencia); el segundo (diagnóstico) no es significativo pero sí la combinación simultánea de los niveles de ambos factores (interacción): $\mathrm{F}=2,57$; gl 4, 52; sig $=0,0489$. La prueba de Scheffé es significativa en los pacientes de la Unidad de Rehabilitación frente al resto. Esto confirmaría que la dependencia institucional tiende a reducir el estado funcional del enfermo. No es significativa la diferencia en el estado funcional respecto a sexo ni edad.

Tabla 2

Estado funcional medio por lugar de residencia y diagnóstico

\begin{tabular}{lcrrrrrrrr}
\hline & \multicolumn{8}{c}{ Valoración media estado funcional según lugar } \\
\multicolumn{1}{c}{ Diagnóstico } & U. Rehabilitación & \multicolumn{2}{c}{ Capital } & \multicolumn{2}{c}{ Provincia } & \multicolumn{1}{c}{ Total media } & Significatividad \\
\hline Residual & 45,56 & $( \pm 7,26)$ & 63,00 & $( \pm 19,87)$ & 45,00 & $( \pm 12,91)$ & 50,28 & $( \pm 14,60)$ & \\
\hline Paranoide & 46,00 & $( \pm 16,73)$ & 70,00 & $( \pm 11,55)$ & 59,38 & $( \pm 13,60)$ & 59,61 & $( \pm 15,10)$ & - \\
\hline Otra & 35,20 & $( \pm 8,67)$ & 65,00 & $( \pm 7,07)$ & 73,33 & $( \pm 15,27)$ & 52,60 & $( \pm 20,89)$ & \\
\hline Total media & $42,95^{*}$ & $( \pm 11,18)$ & 66,79 & $( \pm 14,09)$ & 58,82 & $( \pm 14,99)$ & & & Interacción \\
\hline Significatividad & \multicolumn{10}{c}{$<0,001$} & & & & 0,0489 \\
\hline
\end{tabular}

* Significatividad de prueba post-hoc frente al resto de grupos.

El $80 \%$ de los pacientes están solteros. El 10\% casados y el resto divorciados o separados. El $20 \%$ tienen hijos. Su cuidador principal, en los pacientes que residen fuera de la Unidad de Rehabilitación es la madre (37\%), seguido de la hermana (20\%). Dada la edad media del paciente, la madre forma parte de la tercera edad. El $87 \%$ de éstas residen en la provincia, lo que incrementa notablemente la dificultad de los cuidados.

\section{2) Perfil de Calidad de Vida Lancashire-EU}

Respecto a las relaciones laborales, un $12 \%$ de los pacientes tiene trabajo en sentido estricto, en tareas de limpieza (2 pacientes), agricultura (2), ayuda a domicilio (1), hostelería (1) e industria (1). La valoración media que hacen de sus ocupaciones es de 5,94 $( \pm 0,96)$, en una escala del 1 al 7 de satisfacción vital (ESV). El resto de la información del ítem relacionado con el trabajo no era consistente, por lo que se decide no incluirla en la siguiente fase del análisis.

La media final en la Escala de Satisfacción Vital (LSS): es de 4,58 $( \pm 0,83)$. Según los distintos dominios, vemos que la valoración subjetiva media que hacen, ordenados de mayor a menor satisfacción es: seguridad 5,11 $( \pm 1,40)$; familia 4,77 
$( \pm 1,54)$; religión $4,76( \pm 1,62)$; ocio $4,58( \pm 1,18)$; alojamiento $4,52( \pm 0,99)$; salud $4,51( \pm 1,23)$; relaciones sociales $4,28( \pm 1,32)$; finanzas $4,11( \pm 1,52)$.

La diferencia en medias es significativa (Tabla 3), agrupando por lugar de residencia, en los ítems religión: ANOVA, F $=6,87$; gl. 2, 58; sig $=0,002$; seguridad (rangos medios): Kruskal-Wallis, $\chi^{2}=10,12$; gl. 2; sig $=0,006$; y familia: ANOVA, $\mathrm{F}=3,17$; gl. 2, 58; sig $=0,05$. Las pruebas post hoc establecen que los pacientes de la Unidad de Rehabilitación frente al resto son los que tienen el mayor grado de satisfacción con la religión, el menor grado con respecto a la seguridad y la familia.

Tabla 3

Indicadores de calidad de vida escala ESV-LSS

\begin{tabular}{|c|c|c|c|c|c|c|c|c|c|c|c|}
\hline \multirow{3}{*}{ Indicador } & \multirow{3}{*}{$\begin{array}{c}\text { Media } \\
(D T) \\
\end{array}$} & \multicolumn{10}{|c|}{ Medias-Mediana particulares significativas } \\
\hline & & \multicolumn{3}{|c|}{ Lugar } & \multicolumn{4}{|c|}{ Sexo } & \multicolumn{3}{|c|}{ Vive solo } \\
\hline & & U. Rehab. & Cap. & Prov. & Sig. & Hombre & Mujer & Sig. & Sí & No & Sig. \\
\hline Ocio & $\begin{array}{c}4,58 \\
( \pm 1,18)\end{array}$ & & & & & & & & & & \\
\hline Religión & $\begin{array}{c}4,76 \\
( \pm 1,62)\end{array}$ & $\begin{array}{c}5,8^{*} \\
( \pm 1,4)\end{array}$ & $\begin{array}{c}4,53 \\
( \pm 1,3)\end{array}$ & $\begin{array}{c}4,2 \\
( \pm 1,6)\end{array}$ & 0,002 & $\begin{array}{c}4,42 \\
( \pm 1,64)\end{array}$ & $\begin{array}{c}5,40 \\
( \pm 1,41)\end{array}$ & 0,024 & & & \\
\hline Finanzas & $\begin{array}{c}4,11 \\
( \pm 1,5)\end{array}$ & & & & & & & & $\begin{array}{c}2,95 \\
( \pm 1,97) \\
\end{array}$ & $\begin{array}{c}4,34 \\
( \pm 1,42)\end{array}$ & 0,007 \\
\hline $\begin{array}{l}\text { Aloja- } \\
\text { miento }\end{array}$ & $\begin{array}{c}4,52 \\
( \pm 0,99)\end{array}$ & & & & & & & & & & \\
\hline Seguridad & $\begin{array}{c}5,11 \\
( \pm 1,40)\end{array}$ & $4,50^{*}$ & 6,00 & 6,00 & 0,006 & & & & & & \\
\hline Familia & $\begin{array}{c}4,77 \\
( \pm 1,54)\end{array}$ & $\begin{array}{c}4,08^{*} \\
( \pm 1,78)\end{array}$ & $\begin{array}{c}5,11 \\
( \pm 1,24)\end{array}$ & $\begin{array}{c}5,08 \\
( \pm 1,38)\end{array}$ & 0,05 & & & & & & \\
\hline Sociedad & $\begin{array}{c}4,28 \\
( \pm 1,32)\end{array}$ & & & & & & & & & & \\
\hline Salud & $\begin{array}{c}4,51 \\
( \pm 1,23)\end{array}$ & & & & & & & & & & \\
\hline
\end{tabular}

* Significatividad prueba post-hoc frente al resto de grupos.

En lo concerniente a las preguntas de valoración objetiva (caso de ser aplicable) de los respectivos ítems, no hay relación significativa en las respuestas respecto de las variables de agrupación, salvo en el lugar de residencia para la pregunta: en el último año, ¿ha habido ocasiones en las que hubiera querido haber 
participado en la vida y decisiones familiares, pero no pudo hacerlo? A este respecto la prueba ji-cuadrado arroja un $\chi^{2}=10,36$; $\mathrm{gl}=2$; sig. $=0,006^{4}$.

La forma de medir el estado afectivo y la autoestima del paciente se realiza mediante dos escalas constituida cada una por 10 preguntas de respuesta categórica. La primera se aplica para conocer cómo es el estado emocional del paciente, valorando la presencia de sentimientos positivos o negativos. Se ha obtenido una media (en el recuento de preguntas afirmativas) de $2,1( \pm 1,44)$ sobre 5 en afectividad positiva y una media de $1,9( \pm 1,69)$ sobre 5 en afectividad negativa. Resulta una valoración media/baja de su estado de ánimo. La segunda escala se refiere a la autoestima o punto de vista que tiene el paciente de sí mismo. Se obtiene una puntuación muy alta de autoestima positiva, con una media de $3,65( \pm 1,67)$ sobre 5 y una puntuación baja de autoestima negativa de $2,04( \pm 1,73)$ sobre 5 , por lo que se concluye que el paciente tiene un alto concepto de sí mismo.

Se repite el procedimiento comparativo por grupos, para las variables relacionadas con la afectividad y la autoestima (positivas-negativas). No se encuentran diferencias significativas en relación a ninguna variable de agrupación, lo que indica que todos los pacientes tienen una apreciación similar con respecto a sus emociones y consideración de sí mismos.

El estudio analiza, a continuación, la apreciación que el paciente percibe sobre su calidad de vida global y se confronta con la observada por el entrevistador. Se utiliza, en el primer caso, la media de todas las valoraciones (excepto ítem del trabajo) de la Escala de Satisfacción Vital propuestas en el cuestionario (ESVLSS Life Satisfation Scale) y en el segundo caso, la Uniescala (calidad de vida objetivada por el entrevistador) que califica también globalmente la vida del paciente de 1 a 10. Unificando escalas a una centesimal, en media, diferían notablemente una y otra. Así la valoración subjetiva media del paciente fue de 65 $( \pm 12)$, mientras que la del entrevistador fue de $44( \pm 19)$.

En esta última (Uniescala) existe diferencia contrastada: $A N O V A, F=9 ; \mathrm{gl}$ 2, 58; sig. < 0,001; la prueba de Scheffé establece la significatividad en los sujetos que residen en la capital con respecto a los otros dos grupos; poniéndose de manifiesto (para el entrevistador) una peor calidad de vida en los residentes de la Unidad de Rehabilitación y en la provincia (por este orden). La valoración subjetiva es igual en media para todos los pacientes según todos los criterios de agrupación (lugar de residencia, diagnóstico, sexo, si vive solo y relación con los Servicios de Salud Mental). Sin embargo, en la diferencia de valoración ${ }^{5}$ (subjeti-

4 Hay una diferencia (positiva) significativa entre la frecuencia observada y la teórica en el caso de los residentes en la Unidad de Rehabilitación para la respuesta afirmativa y en los residentes de la capital para la negativa.

En la Unidad de Rehabilitación todos los pacientes tienen una valoración subjetiva superior a la objetivada. En el caso de los residentes en la capital, 3 pacientes tienen una valoración inferior y en la provincia 1. Así, la variable Diferencia sólo se refiere a los 57 casos en los que la valoración subjetiva es superior a la objetivada. 
va-objetivada), sí es significativa para los residentes de la Unidad de Rehabilitación frente al resto de grupos: ANOVA, F = 5,40; gl. 2, 54; sig = 0,007. Es en estos pacientes en los que existe mayor discrepancia entre la apreciación que hace el observador y la que ellos mismos hacen (Tabla 4). Respecto al resto de variables de agrupación, no existen diferencias en este caso.

Tabla 4

Valoraciones medias y diferencia de valoración

\begin{tabular}{|c|c|c|c|c|}
\hline & \multicolumn{4}{|c|}{ Valoración media según lugar } \\
\hline & U. Rehabilitación & Capital & Provincia & Significatividad \\
\hline$E S V-L S S$ & $\begin{array}{c}65,81 \\
( \pm 12,84)\end{array}$ & $\begin{array}{c}68,51 \\
( \pm 9,59)\end{array}$ & $\begin{array}{c}63,74 \\
( \pm 12,26)\end{array}$ & - \\
\hline Uniescala & $\begin{array}{c}33,95 \\
( \pm 15,60)\end{array}$ & $\begin{array}{c}58,64^{*} \\
( \pm 15,75)\end{array}$ & $\begin{array}{c}43,57 \\
( \pm 17,54)\end{array}$ & $<0,001$ \\
\hline Diferencia & $\begin{array}{c}31,86^{*} \\
( \pm 18,35)\end{array}$ & $\begin{array}{c}15,13 \\
( \pm 11,49)\end{array}$ & $\begin{array}{c}20,98 \\
( \pm 12,35)\end{array}$ & 0,007 \\
\hline
\end{tabular}

* Significatividad prueba post-hoc frente al resto de grupos.

Estado funcional y calidad de vida objetivada por el entrevistador (Uniescala) arroja un coeficiente de correlación de Pearson 0,84 (sig bilateral $<0,001)$. Son dos variables marcadamente relacionadas y medidas de forma independiente. Estado funcional y calidad de vida subjetiva escala ESV-LSS (autovaloración global) arroja un coeficiente de correlación 0,37 (sig bilateral $=0,003$ ). Consecuentemente, existe una correlación entre el estado funcional y la diferencia de valoración de $-0,63$ (sig bilateral $<0,001$ ). Son los pacientes con mayor valoración en la escala EEAG los que presentan menor discrepancia en ambas valoraciones (Tabla 5).

Tabla 5

Correlaciones

\begin{tabular}{ccc}
\hline & $\begin{array}{c}\text { Estado } \\
\text { funcional }\end{array}$ & Significatividad \\
\hline ESV-LSS & 0,37 & 0,003 \\
\hline Uniescala & 0,84 & $<0,001$ \\
\hline Diferencia & $-0,63$ & $<0,001$ \\
\hline
\end{tabular}


Pensamos que es el estado funcional del paciente el que determinará el nivel de calidad de vida evaluada por el observador. Es decir, con un nivel más alto en la Escala de Evaluación de la Actividad Global, el paciente podrá tener mejores condiciones de vida: cuantas más cosas pueda hacer, mayores oportunidades él mismo se dará. Esto se infiere en la valoración hecha por el entrevistador, porque respecto a la propia evaluación, la influencia es menos importante.

En referencia a los aspectos que mejorarían la calidad de vida de los pacientes y a tenor de lo contestado en primer lugar tenemos que: El dinero, la salud, el hogar y la pareja acaparan el $62 \%$ de la opinión sobre mejora de sus condiciones personales. No existen diferencias significativas entre los grupos según residencia, sexo, edad ni diagnóstico.

\section{Discusión}

Sabemos, por la práctica diaria y por los resultados de otros estudios, que la familia se ha convertido en una importante fuente de cuidados informales para estas personas. En el estudio sobre Calidad de Vida llevado a cabo por Gaite y colaboradores (Santander) en 2002 (16), casi las tres cuartas partes de los pacientes esquizofrénicos incluidos, vivían con sus familiares. Pero en él se seleccionaron pacientes con edades comprendidas entre los 18 y los 65 años, eliminando aquellos con un grado alto de institucionalización.

En el nuestro, con una población mayor en edad y otros criterios de selección, lo que resulta llamativo es la proporción (31\%) de pacientes que residen en la Unidad de Rehabilitación. Si examinamos entre las posibles causas, vemos que la mayoría nunca llegó a integrarse en la comunidad por carecer de cuidadores y permanecen institucionalizados desde antes de la reforma. Los que han ingresado en los últimos años (pacientes que no pueden vivir autónomos) lo han hecho, principalmente, porque sus cuidadores han fallecido o no pueden seguir con esa función por más tiempo.

La Calidad de Vida ha sido estudiada aplicando el LQoLP, cuyas características psicométricas y su aplicabilidad fue validado en el estudio EPSILON (14). Este instrumento integra tanto indicadores objetivos como subjetivos de Calidad de Vida. Sigue estando sin resolver la concordancia existente entre estos dos indicadores. Algunos autores opinan (17) que los factores subjetivos de Calidad de Vida, no reflejan bien las condiciones ambientales. Mientras otros $(18 ; 19)$ dicen que sí existe concordancia entre estos dos aspectos, sobre todo en pacientes con una buena capacidad de introspección. En nuestro estudio, hemos podido encontrar evidencias en ambos sentidos. Son los pacientes de unidad residencial los que mayor discrepancia presentan ante ambos tipos de valoración. Asimismo existe 
correlación inversa entre esa discrepancia (subjetiva-objetiva) y el estado funcional. Es decir, a mayor estado funcional en los pacientes (con independencia incluso del lugar de residencia), menor discrepancia en ambas valoraciones.

El término calidad de vida, sin embargo, no está exento de controversia. Según los criterios establecidos por la UNESCO en 1979, la calidad de vida comprende todos los aspectos de las condiciones de vida de los individuos, es decir, todas sus necesidades y la medida en que se satisfacen. Incluiría, por tanto, ámbitos materiales e inmateriales de la vida que se desarrolla en un medio concreto. Es este un enfoque principalmente subjetivo, ya que se presenta en torno a la necesidad.

También podríamos definir la calidad de vida en palabras de Amartya Sen (20), como un compendio de capacidades y funcionamientos que delimitan las oportunidades y ventajas personales para funcionar en una sociedad concreta. Sen se refiere a los funcionamientos como representantes del estado de una persona: las cosas que logra hacer o ser al vivir. Las capacidades de una persona reflejan la oportunidad de elegir y la posibilidad de adaptarse a los cambios.

La noción sobre calidad de vida no es únicamente autopercepción del estado en que un individuo se encuentra. También puede ser entendida externamente. Es por lo que hemos aplicado un doble enfoque a lo largo de nuestro análisis: autoevaluación y evaluación por observador exterior.

Las distintas pruebas estadísticas realizadas, nos permiten concluir que son dos las variables que afectan a la calidad de vida objetivada por el entrevistador de los pacientes incluidos en el estudio: estado funcional y lugar de residencia. A su vez, el lugar de residencia, repercute de manera influyente en el estado funcional de los pacientes. Cobrando relevancia la dependencia o no institucional en la calidad de vida objetivada por el entrevistador. Sin embargo, respecto a la percepción global media (grado de satisfacción) que los pacientes tienen sobre su propio bienestar, no se ve influida por ninguna variable de referencia, salvo de nuevo (aunque de forma menos relevante), por el estado funcional. En este sentido y con carácter general, los pacientes tienen un marcado convencimiento de que sus vidas presentan una buena calidad, como decíamos, con independencia del lugar de residencia (particularmente, vimos en la Tabla 3, discrepancias por grupos). ¿Qué valoración debe prevalecer?

Creemos que ambos criterios no son mutuamente excluyentes sino que se complementan. El subjetivo nos da cuenta de la percepción que sobre su vida tiene el enfermo y del grado de satisfacción. El del observador se basa en las facultades que la sociedad actual entiende, debería tener el paciente para su desarrollo personal dentro de la misma.

El grado relativamente alto de satisfacción subjetiva que presentan los pacientes institucionalizados, podría explicarse, como señalan Leisse y Kallert (7), porque 
evalúan su calidad de vida a partir de sus necesidades y propósitos, que se reducen durante el progreso de la enfermedad, sobre todo con los síntomas negativos y la hospitalización. Por otra parte, el entendimiento sobre sus propias facultades, se ve condicionado porque las personas con las que compararse son otros pacientes como ellos.

La diferencia de valoración (subjetiva-objetiva) es significativamente mayor en los pacientes bajo dependencia institucional. En el otro extremo, los enfermos residentes en la capital (y en menor medida los de la provincia) son quienes presentan mayor concordancia en ambos tipos de valoración y según esto, los que presentarían mayores posibilidades de desarrollo.

Las acciones subsiguientes deberían ir encaminadas a que cada paciente, al menos, pueda vivir con arreglo al estado funcional que le permita su enfermedad. Y en la medida de lo posible, hacer que su estado funcional también mejore. Estas medidas, entendemos, incrementarán o al menos impedirán un deterioro de su calidad de vida, con independencia de cómo tratemos de medirla.

\section{Agradecimientos}

Este artículo ha sido realizado a partir del trabajo de campo del estudio: Esquizofrenia, 20 años de desinstitucionalización en Segovia; financiado por la Consejería de Sanidad de la Junta de Castilla y León, junto a otros seis proyectos de investigación en materia de coordinación sociosanitaria, (publicación BOCyL de 15-2-2005). Investigadores de aquel estudio fueron: Isabel Criado Martín (coordinadora de la investigación); Rafael Acebes Valentín; Concha Covarrubias Fernández y Sonia Lafuente Lázaro.

\section{BIBLIOGRAFÍA}

(1) Desviat, M., La Reforma Psiquiátrica, Madrid, DOR, 1994.

(2) ARIAS, P., y otros, «Rehabilitación Psicosocial del trastorno mental severo. Situación actual y recomendaciones», AEN, 2002, Cuadernos técnicos, 6, pp. 15-18.

(3) Bobes, G. J., y otros, Calidad de Vida en las esquizofrenias, Barcelona, J. R. Prous Editores, 1995.

(4) Angermeyer, M. C.; Kilian, R., «Theoretical Models of Quality of Life for Mental Disoders», en Katschnig, R., y otros (eds.), Quality of Life in Mental Disorders, Nueva York, Wiley, 1997, pp. 19-30.

(5) BARrY, M. M.; ZISSI, A., «Quality of Life as an Outcome Measure in Evaluating Health Services: a Review of the Empirical Evidence», Soc. Psychiatr. Epidemiol., 1997, 32, pp. 38-47.

(6) Katschnig, H.; KönIG, P. (eds.), Schizophrenia and Quality of Life, Viena, Springer, 1997.

(7) LeISSE, M.; KalleRT, T. W., «Social Integration and the Quality of Life of Schizophrenic Patients in Different Types of Complementary Care», Eur. Psychiatry, 2000, 15, pp. 450- 460. 
(8) BJÖRKMAN, T.; HANSSON, L., «Predictores de la mejoría en la calidad de vida de individuos enfermos mentales a largo plazo que recibieron gestión de casos», Eur. Psychiatry, 2002, ed. española, 9, pp. 283-290.

(9) Sen, A. K., «Capacidad y bienestar», en Nussbaum, M.; Sen, A. K. (eds.), La calidad de vida, Méjico, F.C.E., 1996.

(10) CIE-10, Clasificación de los trastornos mentales y del comportamiento, Madrid, Panamericana, 2000.

(11) Asociación Americana de Psiquiatría, Manual diagnóstico y estadístico de los trastornos mentales, Barcelona, Masson, 2002.

(12) Bobes, G. J., y otros, Banco de instrumentos básicos para la práctica de la psiquiatría clínica, Barcelona, Ars Médica.

(13) FERnÁNDEZ RodrígueZ, J.; YÁÑIz IgaL, B., Instrumentos de evaluación de la calidad de vida en las esquizofrenias, 2002.

(14) GaIte, L., y otros, «Quality of Life in Schizophrenia: Development, Reliability and Internal Consistency of the Lancashire Quality of Life Profile-European Version: EPSILON Study 8», Br. J. Psychiatry, 2000, 177(39), pp. 4-54.

(15) YoshioKA, K., Kyplot 2.0 Beta 15, 1997-2001, www.woundedmoon.org /win32 /kyplot.html

(16) Gaite, L., y otros, «Quality of Life in Patients with Schizophrenia in Five European Countries: the EPSILON study», Acta Psychiatr. Scand., 2002, 105(4), pp. 283-292.

(17) AtKinson, M.; Saharon, Z.; Chuang, H., «Characterizing Quality of Life among Patients with Chronic Mental Illnes: a Critical Examination of the Self-report Methodology», Am. J. Psychiatry, 1997, 154, pp. 99-105.

(18) Doyle, M.; Flanagan, S.; Browne, S., y otros, «Subjective and External Assessments of Quality of Life in Schizofhrenia: Relationship to Insight», Acta Psychiatr. Scand., 1999, 99, pp. 466-472.

(19) Lobana, A., y otros, «Quality of Life in Schizophrenia in India: Comparison of Three Approaches», Acta Psychiatr. Scand., 2001, 104, pp. 51-55.

* Sonia Lafuente Lázaro, psiquiatra. Hospital General de Segovia; José Santos Francisco González, Licenciado en Economía Cuantitativa; Isabel Criado Martín, Enfermera de Salud Mental; Concha Covarrubias Fernández, Enfermera Especialista de Salud Mental. Hospital General de Segovia.

Correspondencia: suylafuente@ono.com

** Fecha de recepción: 8-III-2007. 